\title{
Accelerating Universe with Viscous Cosmic String in Quadratic Form of Teleparallel Gravity
}

\author{
S. R. Bhoyar ${ }^{1 *}$, V. R. Chirde ${ }^{2}$, S. H. Shekh ${ }^{3}$ \\ ${ }^{1}$ Department of Mathematics, Phulsing, Naik Mahavdyalaya, Pusad-445216, India \\ ${ }^{2}$ Department of Mathematics, G. S. G. Mahavidyalaya, Umarkhed-445206, India \\ ${ }^{3}$ Department of Mathematics, S.P.M Science and Gilani Arts and Commerce College-Ghatanji- \\ 445301, Yavatmal, Maharashtra, India
}

Received 6 December 2018, accepted in final revised form 2 February 2019

\begin{abstract}
In this paper, we have investigated Kantowaski-Sachs cosmological model with bulk viscous and cosmic string in the framework of Teleparallel Gravity so called $f(T)$ gravity, where $T$ denotes the torsion scalar. The behavior of accelerating universe is discussed towards the particular choice of $f(T)=\alpha T+\beta T^{m}$. The exact solutions of the field equations are obtained by applying variable deceleration parameter which is linear in time with a negative slope. The physical behavior of these models has been discussed using some physical quantities. Also, the function of the torsion scalar for the universe is evaluated.
\end{abstract}

Keywords: Kantowaski-Sachs model; Bulk viscous and cosmic string; $f(T)$ gravity.

() 2019 JSR Publications. ISSN: 2070-0237 (Print); 2070-0245 (Online). All rights reserved. doi: http://dx.doi.org/10.3329/jsr.v11i3.39220 J. Sci. Res. 11 (3), 249-262 (2019)

\section{Introduction}

An awesome abundance of observational evidence in favor the late-time accelerating expansion does not fit within the framework of General Relativity (GR). The proposals that have been put forward to explain this observed phenomenon can basically be classified into two categories. First, an exotic component with negative pressure called mysterious energy or Dark Energy (DE) introduce in to Einstein's general relativity and second is by changing the gravity law through the modification of action in GR.

Among the various modifications of GR, $f(R)$ gravity where the gravitational Lagrangian is given by an arbitrary function of the Ricci scalar, attracting more and more attention during the last decade. A complete review on $f(R)$ gravity is given by Copeland

\footnotetext{
Corresponding author: sbhoyar68@yahoo.com
} 
et al. [1], Chiba et al. [2], Nojiri and Odintsov [3,4]. Another modification of standard GR is $f(R, T)$ gravity where the gravitational Lagrangian is given by an arbitrary function of the Ricci scalar and the trace of the stress energy tensor [5]. Using this theory Harko et al. [6] have discussed several aspects of this theory including FRW dust universe. Sharif and Zubair [7] found that the picture of equilibrium thermodynamics is not feasible in $f(R, T)$ gravity even if we specify the energy density and pressure of dark components thus the non-equilibrium treatment is used to study the laws of thermodynamics in both forms of the energy momentum tensor of dark components. Katore et al. [8] investigated some cosmological model with DE source in $f(R, T)$ gravity. Chandel and Ram [9] generated new classes of solutions of field equations starting from known solutions for an anisotropic Bianchi type-III cosmological model with perfect fluid in $f(R, T)$ gravity. Chaubey et al. [10] has obtained a new class of Bianchi type cosmological models in $f(R, T)$ gravity, while, Sahoo et al. [11] investigated an axially symmetric space-time in the presence of a perfect fluid source within the frame work of $f(R, T)$ gravity. Recently, Bhoyar et al. [12,13], Chirde and Shekh [14,15] have investigated some cosmological models with some fluids within the frame work of $f(R, T)$ gravity.

Another alternative goes back to 1928 with Einstein's attempt to unify gravity and electromagnetism through the introduction of a tetrad (vierbein) field, with the concept of absolute parallelism or teleparallelism [16] known as Tele parallel Gravity (TG) or $f(T)$ gravity. The gravitational field equation of TG is described in terms of torsion instead of curvature [17]. An advantage of $f(T)$ theory is that its field equation is only second order. Various aspects of $f(T)$ theory have been investigated in the references [18-20]. Jamil et al. [21] tried to resolve the Dark Matter (DM) problem in the light of $f(T)$ gravity and successfully obtained the flat rotation curves of galaxies containing DM as component with the density profile of DM in galaxies. Also gives the interacting DE model in the framework of same theory for a particular choice of $f(T)$. Particle creation in flat Friedman Robertson Walker universe in the framework of $f(T)$ gravity was investigated by Setare and Houndjo [22]. Chirde and Shekh [23-25] investigated some cosmological model in $f(T)$ gravity. Very recently, Bhoyar et al. [26] discussed stability of accelerating Universe with linear equation of state in $f(T)$ gravity using hybrid expansion law. Aygün et al. [27] investigated Teleparallel energy-momentum distribution of various black hole and wormhole metrics.

According to the prediction of grand unified theory (GUT), after the Big-bang the universe may have undergone a series of phase transitions as its temperature lowered below some critical temperature [28]. It can provide grow to topologically established defects such as strings, domain walls, and monopoles. Surrounded by these cosmological structures, cosmic strings are the most incredible consequences [29], because it gives rise to density perturbations, which might have been found during a phase transition in the early universe which lead to formation of galaxies [30]. The study of gravitational effects from such strings will be interesting because of the cosmic strings coupled stress-energy to the gravitational field. The treatment of strings in GR (in view of massive strings as the geometric strings (massless) with particles attached along their expansions) has been 
initially given by Letelier [31]. Along with sahoo [32] investigated Cosmic Strings Coupled with Maxwell Fields in Bimetric Relativity on Kantowski-Sachs space-time. Mahanta et al. [33] discussed about the bulk viscous string cloud with strange quark matter in Brans-Dicke theory. Sahoo et al. [34] talk over Bianchi Type-III String Cosmological Model with bulk viscous fluid in Lyra Geometry.

Most of the researchers assume cosmological models with perfect fluid or pressure less (dust) distribution but to have realistic models we should consider the presence of a material distribution other than a perfect fluid. At the early phase evolution of the universe when neutrino decoupling occurred, the matter behaved like a viscous fluid (viscosity mechanism in cosmology can account for high entropy per baryon in the present universe). Also, the viscosity is associated with GUT phase transition and string creation. Santos et al. [35] have derived exact solution of field equations by considering, viscous coefficient as a power function of mass density. Wang [36] have discussed cloud string with bulk viscosity within the frame work of LRS Bianchi type-I and Kantowski-Sachs string model. Integrability of the cosmic string with bulk viscous fluid had investigated by Yadav et al. [37].

\section{2. $f(\mathrm{~T})$ Gravity Formalism}

In this section we give a brief description of the $f(T)$ model and a detailed derivation of its field equations.

Let us define the notations of the Latin subscript as these related to the tetrad field and the greek one related to the space-time co-ordinates. For a general space-time metric, we can define the line element as

$$
d S^{2}=g_{\mu \nu} d x^{\mu} d x^{v}
$$

This line element can be converted to the Minkowski's description of the transformation called tetrad, as follows

$$
\begin{aligned}
& d S^{2}=g_{\mu \nu} d x^{\mu} d x^{v}=\eta_{i j} \theta^{i} \theta^{j} \\
& d x^{\mu}=e_{i}^{\mu} \theta^{i}, \theta^{i}=e_{\mu}^{i} d x^{\mu},
\end{aligned}
$$

where $\eta_{i j}$ is a metric on Minkowski space-time and $\eta_{i j}=\operatorname{diag}[1,-1,-1,,-1]$ and

$$
e_{i}^{\mu} e_{\mu}^{i}=\delta_{v}^{\mu} \text { or } e_{i}^{\mu} e_{\mu}^{j}=\delta_{i}^{j}
$$

The root of metric determinant is given by $\sqrt{-g}=\operatorname{det}\left[e_{\mu}^{i}\right]=e$. For a manifold in which the Riemann tensor part without the torsion terms is null (contribution of the LeviCivita connection) and only the non-zero torsion terms exist, the Weitzenbocks connection components are defined as

$$
\Gamma_{\mu v}^{\alpha}=e_{i}^{\alpha} \partial_{\nu} e_{\mu}^{i}=-e_{\mu}^{i} \partial_{v} e_{i}^{\alpha} .
$$

which has a zero curvature but nonzero torsion. Through the connection, we can define the components of the torsion tensors as 


$$
T_{\mu v}^{\alpha}=\Gamma_{\mu v}^{\alpha}-\Gamma_{v \mu}^{\alpha}=e_{i}^{\alpha}\left(\partial_{\mu} e_{v}^{i}-\partial_{\mu} e_{\mu}^{i}\right)
$$

The difference between the Levi-Civita and Weitzenbock connections is a space-time tensor, and is known as the contortion tensor:

$$
K_{\alpha}^{\mu v}=\left(-\frac{1}{2}\right)\left(T_{\alpha}^{\mu v}+T_{\alpha}^{v \mu}-T_{\alpha}^{\mu v}\right)
$$

For facilitating the description of the Lagrangian and the equations of motion, we can define another tensor $S_{\alpha}^{\mu \nu}$ from the components of the torsion and contortion tensors, as

$$
S_{\alpha}^{\mu v}=\left(\frac{1}{2}\right)\left(K_{\alpha}^{\mu v}+\delta_{\alpha}^{\mu} T_{\beta}^{\beta v}-\delta_{\alpha}^{v} T_{\beta}^{\beta \mu}\right)
$$

The torsion scalar is

$$
T=T_{\mu v}^{\alpha} S_{\alpha}^{\mu v}
$$

Now, we define the action by generalizing the Teleparallel Theory i.e. $f(T)$ theory as

$$
S=\int\left[T+f(T)+L_{\text {matter }}\right] e d^{4} x
$$

Here, $f(T)$ denotes an algebraic function of the torsion scalar. Making the functional variation of the action (9) with respect to the tetrads, we get the following equations of motion

$$
S_{\mu}^{v \rho} \partial_{\rho} T f_{T T}+\left[e^{-1} e_{\mu}^{i} \partial_{\rho}\left(e e_{i}^{\alpha} S_{\alpha}^{\nu \rho}\right)+T^{\alpha}{ }_{\lambda \mu} S_{\alpha}^{\nu \lambda}\right]\left(1+f_{T}\right)+\frac{1}{4} \delta_{\mu}^{v}(T+f)=4 \pi T_{\mu}^{v}
$$

The field equation (10) is written in terms of the tetrad and partial derivatives and appears very different from Einstein's equations.

where $T_{\mu}^{v}$ is the energy momentum tensor, $f_{T}=d f(T) / d T$ and by setting $f(T)=a_{0}=$ constant this is dynamically equivalent to the GR.

\section{Metric, Kinematical Parameters and Field Equations}

The line element of homogeneous anisotropic Kantowaski-Sachs space-time is given by

$$
d s^{2}=d t^{2}-A^{2} d x^{2}-B^{2}\left(d y^{2}+d z^{2}\right)
$$

where the metric potentials $A$ and $B$ be the functions of time $t$ only.

The corresponding Torsion scalar is given by

$$
T=-2\left(2 \frac{\dot{A}}{A} \frac{\dot{B}}{B}+\frac{\dot{B}^{2}}{B^{2}}\right)
$$

Let us consider the matter content energy-momentum tensor of a bulk viscous fluid containing one dimensional cosmic string as

$$
T_{\mu}^{v}=\rho u^{v} u_{\mu}-\lambda x_{\mu} x^{v}-\xi \theta\left(u^{v} u_{\mu}+g_{\mu}^{v}\right)
$$


here is the rest energy density for a cloud of massive strings with particle attached along its extension given by $\rho$

$$
\rho=\rho_{p}+\lambda
$$

$\rho_{p}$ being the particle energy density, $\lambda$ the string tension density may be positive or negative, $u^{v}$ the four velocity for the cloud of particles and $x^{v}$ the four vector which represents the strings direction. Thus we have,

$$
\begin{aligned}
& u^{v} u_{v}=-1=-x^{v} x_{v} \text { and } \\
& u_{v} x^{v}=0,
\end{aligned}
$$

together with commoving co-ordinates

$$
u^{v}=(0,0,0,1) \text { and } x^{v}=\left(\frac{1}{A}, 0,0,0\right),
$$

From the equation of motion (10), Kantowaski-Sachs space-time (11) for the fluid of stress energy tensor (13) can be written as

$$
\begin{aligned}
& (T+f)+4\left(1+f_{T}\right)\left\{\frac{\ddot{B}}{B}+\frac{\dot{B}^{2}}{B^{2}}+\frac{\dot{A}}{A} \frac{\dot{B}}{B}\right\}+4 \frac{\dot{B}}{B} \dot{T} f_{T T}=k^{2}(\lambda-p+\xi \theta), \\
& (T+f)+2\left(1+f_{T}\right)\left\{\frac{\ddot{A}}{A}+\frac{\ddot{B}}{B}+\frac{\dot{B}^{2}}{B^{2}}+3 \frac{\dot{A}}{A} \frac{\dot{B}}{B}\right\}+2\left\{\frac{\dot{A}}{A}+\frac{\dot{B}}{B}\right\} \dot{T} f_{T T}=k^{2}(-p+\xi \theta), \\
& (T+f)+4\left(1+f_{T}\right)\left\{\frac{\dot{B}^{2}}{B^{2}}+2 \frac{\dot{A}}{A} \frac{\dot{B}}{B}\right\}=k^{2}(\rho) .
\end{aligned}
$$

where the $\operatorname{dot}(\cdot)$ denotes the derivative with respect to time $t$.

We define average scale factor and volume respectively as

$$
V=A B^{2}
$$

The generalized mean Hubble parameter which expresses the expansion rate of the space-time, can be given as

$$
H=\frac{1}{3}\left(H_{1}+H_{2}+H_{3}\right)
$$

where $H_{1}, H_{2}, H_{3}$ are the directional Hubble parameter in the direction of $\mathrm{x}, \mathrm{y}$, and z-axis respectively.

To discussed whether the models either approach isotropy or not, we define an anisotropy parameter of the expansion as

$$
A_{m}=\frac{1}{3} \sum_{i=1}^{3}\left(\frac{H_{i}-H}{H}\right)^{2} .
$$

The expansion scalar and shear scalar respectively are defined as follows 


$$
\begin{aligned}
& \theta=u_{; \mu}^{\mu}=\frac{\dot{A}}{A}+\frac{\dot{B}}{B}+\frac{\dot{C}}{C}, \\
& \sigma^{2}=\frac{3}{2} H^{2} A_{m} .
\end{aligned}
$$

Another important dimensionless kinematic quantity is the deceleration parameter (DP), which shows whether the universe exhibits accelerating volumetric expansion or not:

$$
q=-1+\frac{d}{d H}\left(\frac{1}{H}\right),
$$

For $-1 \leq q<0, q>0 \& q=0$, the universe exhibit accelerating volumetric expansion, decelerating volumetric expansion and constant-rate volumetric expansion respectively.

\subsection{Solution of field equation}

For any physically relevant model, Hubble parameter and deceleration parameter are the most important observational quantities in cosmology. During 1960s and 1970s, redshift magnitude test has made sure the history about DP which lies between $0 \leq q \leq 1$ and draw very categorical conclusion that the universe is decelerating. Soon after Berman and Gomide in 1983, 1988 get value $q \geq-1$, and since $-1 \leq q \leq 0$ corresponds to accelerating expansion in his proposed a law of variation for Hubble parameter, but the recent theoretical analysis of SNe-Ia surveys, LSS and CMBA spectrum strongly indicate that our Universe is spatially flat and has a phase transition i.e. past deceleration to recent acceleration. So, In order to match the results with this observation, many authors have define different types of solutions (corresponds to DP and scale factor). Chirde and Shekh [25] offered the DP, which is linear in time with a negative slope as,

$$
q=-\frac{\ddot{R} R}{\dot{R}^{2}}=-1+\frac{d}{d t}\left(\frac{1}{H}\right),
$$

this equation can be integrated to give the average scale factor as

$$
R=\exp \int \frac{d t}{\left[\int(1+q) d t+\gamma\right]},
$$

where $\gamma$ be the arbitrary constant.

For the possible explicit determination of $R$, we have to integrate above equation (26). Depending on the choice for the values of deceleration parameter $q$, there are two different ways to integrate

(i) According to Berman $q$ is taken to be a constant either positive or negative which provides an explicit function of $R$ and

(ii) According to new law $q$ is taken to vary with cosmic time for an explicit determination of $R$ which leads to a possible choice of $q$ as 


$$
q=-\frac{a}{t^{2}}+(b-1)
$$

which is the main ansatz of the paper. Here $a>0$ is a parameter having the dimension of square of time and $b$ is a dimensionless constant.

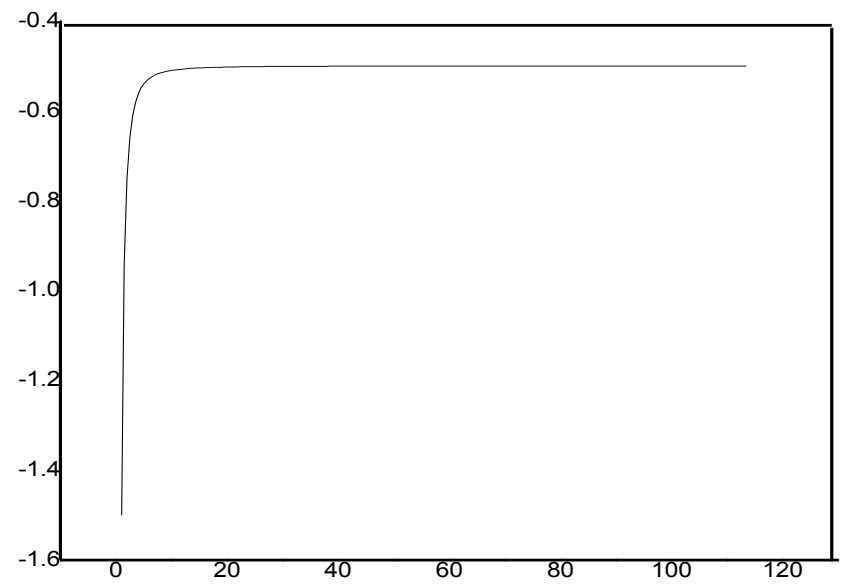

Fig. 1. Deceleration parameter versus time.

Obviously, the different values of $a$ and $b$ will give rise to different models. For $b \geq 1$ corresponds to a decelerating model, for $0<b<1$ it corresponds to standard accelerating model.

Equation (27) can be integrated to give the time variation of the scale factor as

$$
R=\exp \left\{\frac{1}{\beta} \int\left(\frac{t d t}{t^{2}+t \frac{\delta}{b}+\frac{a}{b}}\right)\right\},
$$

The integral appearing in (28) cannot be evaluated for arbitrary values of the constants. Setting $\delta=0$ in (28) and integrating we obtain the average scale factor as

$$
R=\left(t^{2}+\frac{a}{b}\right)^{1 / 2 b},
$$

For this model, the corresponding metric coefficients $A$ and $B$ comes out to be

$$
\begin{aligned}
& A=\left(t^{2}+\frac{a}{b}\right)^{3 n / 2 b(n+2),}, \\
& B=\left(t^{2}+\frac{a}{b}\right)^{3 / 2 b(n+2)} .
\end{aligned}
$$

Using the values of metric coefficients, Kantowaski-Sachs space-time with bulk viscous and cosmic string in the framework of $f(T)$ gravity takes the form 


$$
d s^{2}=d t^{2}-\left(t^{2}+\frac{a}{b}\right)^{6 / 2 b(n+2)} d r^{2}-\left(t^{2}+\frac{a}{b}\right)^{6 / 2 b(n+2)}\left(d \theta^{2}+\sin ^{2} \theta d \phi^{2}\right) .
$$

It is observed that the model hag no singularity and expands with time.

\subsection{Physical parameters}

Energy density and pressure of the universe becomes

$$
\begin{aligned}
& k^{2} \rho=\frac{(1-\alpha) p t^{2}}{\left(t^{2}+a / b\right)}+\frac{(1-2 m) \beta p^{m} t^{2 m}}{\left(t^{2}+a / b\right)^{2 m}}, \\
& k^{2} p=\frac{-\alpha_{1}}{b^{2}(n+2)\left(t^{2}+a / b\right)^{2}}+\frac{-\alpha_{2}}{b^{2}(n+2)\left(t^{2}+a / b\right)^{2 m}}+\frac{3 k^{2} \xi t}{b\left(t^{2}+a / b\right)} .
\end{aligned}
$$

For the specification of $\xi$ we assume that the fluid obeys an equation of the state of the form

$$
p=\gamma \rho,
$$

where $0 \leq \gamma \leq 1$.

In most of the investigations, bulk viscosity is assumed to be a simple power function of the energy density (see, for examples, Pavon [38], Maartens [39]):

$$
\xi(t)=\xi_{0} \rho^{r},
$$

where $\xi_{0}$ and $r$ are constants.

If $r=1$, Eq. (36) may corresponds to a radiative fluid. However, more realistic models are based on $n$ lying in the regime $0 \leq r \leq 1 / 2$. On using (36) in (34), we obtain the following relation for proper pressure:

$$
k^{2} p=\frac{-\alpha_{1}}{b^{2}(n+2)\left(t^{2}+a / b\right)^{2}}+\frac{-\alpha_{2}}{b^{2}(n+2)\left(t^{2}+a / b\right)^{2 m}}+\frac{3 k^{2} \xi_{0} t \rho^{r}}{b\left(t^{2}+a / b\right)} .
$$

\subsection{Model I: Solution for $\xi(t)=\xi_{0}$}

When $r=0$, equation (36) reduces to $\xi(t)=\xi_{0}=$ constant . Hence in this case Energy density and string tension density leads to

$$
\left.k^{2} \rho=\frac{1}{(1+\gamma)}\left\{\frac{(1-\alpha) \alpha_{0} t^{2}}{\left(t^{2}+a / b\right)^{2}}+\frac{(1-2 m) \alpha_{0}^{2} t^{2 m}}{\left(t^{2}+a / b\right)^{2 m}}-\frac{\alpha_{1}}{b^{2}(n+2)\left(t^{2}+a / b\right)^{2}}-\frac{\alpha_{2}}{b^{2}(n+2)\left(t^{2}+a / b\right)^{2 m}}+\frac{3 k^{2} \xi_{0} t}{b\left(t^{2}+a / b\right.}\right)\right\}
$$




$$
k^{2} \lambda=\frac{2 \alpha \alpha_{3}}{b^{2}(n+2)^{2}\left(t^{2}+a / b\right)^{2}}+\frac{2 \alpha_{3} m \beta \alpha_{0}^{m-1} t^{2 m-2}}{b^{2}(n+2)^{2}\left(t^{2}+a / b\right)^{2 m}}-\frac{4 m(m-1)(1-m) \alpha_{0}^{m-1} t^{2 m-2}}{b(n+2)\left(t^{2}+a / b\right)^{2 m-2}}
$$

\subsection{Model II: Solution for $\xi(t)=\xi_{0} \rho$}

When $r=1$, equation (36) reduces to $\xi(t)=\xi_{0} \rho$. Hence in this case Energy density, particle energy density and string tension density leads to

$$
\begin{aligned}
& k^{2} \rho=\frac{1}{(1+\gamma)}\left\{\left(1+\frac{3 k^{2} \xi_{0} t}{b\left(t^{2}+a / b\right)}\right)\left(\frac{(1-\alpha) \alpha_{0} t^{2}}{\left(t^{2}+a / b\right)^{2}}+\frac{(1-2 m) \alpha_{0}^{2} t^{2 m}}{\left(t^{2}+a / b\right)^{2 m}}\right)-\frac{\alpha_{1}}{b^{2}(n+2)\left(t^{2}+a / b\right)^{2}}-\frac{\alpha_{2}}{b^{2}(n+2)\left(t^{2}+a / b\right)^{2 m}}\right\} \\
& k^{2} \rho_{p}=\frac{1}{(1+\gamma)}\left(1+\frac{3 k^{2} \xi_{0} t}{b\left(t^{2}+a / b\right)}\right)\left(\frac{(1-\alpha) \alpha_{0} t^{2}}{\left(t^{2}+a / b\right)^{2}}+\frac{(1-2 m) \alpha_{0}^{2} t^{2 m}}{\left(t^{2}+a / b\right)^{2 m}}\right)-\frac{\alpha_{1}(n+2)+2 \alpha(1+\gamma)^{2} \alpha_{3}}{b^{2}(n+2)\left(t^{2}+a / b\right)^{2}} \\
& -\frac{\alpha_{2}(n+2)+2(1+\gamma)^{2} \alpha_{3} m \beta \alpha_{0}^{m-1} t^{2 m-2}}{b^{2}(n+2)\left(t^{2}+a / b\right)^{2 m}}+\frac{4 m(1+\gamma)(1-n)(m-1) \alpha_{0}^{m-1} t^{2 m-2}}{b(n+2)\left(t^{2}+a / b\right)^{2 m-2}} . \\
& k^{2} \lambda=\frac{2 \alpha \alpha_{3}}{b^{2}(n+2)^{2}\left(t^{2}+a / b\right)^{2}}+\frac{2 \alpha_{3} m \beta \alpha_{0}^{m-1} t^{2 m-2}}{b^{2}(n+2)^{2}\left(t^{2}+a / b\right)^{2 m}}-\frac{4 m(m-1)(1-m) \alpha_{0}^{m-1} t^{2 m-2}}{b(n+2)\left(t^{2}+a / b\right)^{2 m-2}} .
\end{aligned}
$$

The Torsion scalar becomes

$$
T=\frac{p t^{2}}{\left(t^{2}+\frac{a}{b}\right)^{2}}, \quad \text { where } p=-18(1+2 n) / b^{2}(n+2)^{2} .
$$

\subsection{Kinematical parameters}

The spatial volume becomes

$$
V=\left(t^{2}+\frac{a}{b}\right)^{3 / 2 b}
$$

In our investigations we observed that the spatial volume of the universe starts with a constant volume at $t \rightarrow 0$ and with the revival of time it is expanding and increasing, when $t \rightarrow \infty$ the spatial volume $V \rightarrow \infty$. Thus inflation is possible in the universe. This shows that the universe starts evolving with constant volume and expands with cosmic time.

The mean Hubble parameter and the expansion scalar turn out to be 


$$
\begin{aligned}
& H=\frac{t}{b\left(t^{2}+\frac{a}{b}\right)}, \\
& \theta=\frac{3 t}{b\left(t^{2}+\frac{a}{b}\right)} .
\end{aligned}
$$

From the equations (45) and (46) it is observed that the mean Hubble parameter, expansion scalar are the functions of time, at initially $t \rightarrow 0$ both are zero and constant at infinite expansion as $t \rightarrow \infty$. This shows that the universe is expanding with the increase of cosmic time but the rate of expansion decrease which shows that the universe starts evolving with a constant volume with an infinite rate of expansion. The behavior of Hubble's parameter and expansion scalar of the universe verses cosmic time $t$ is shown in the Fig. 2.

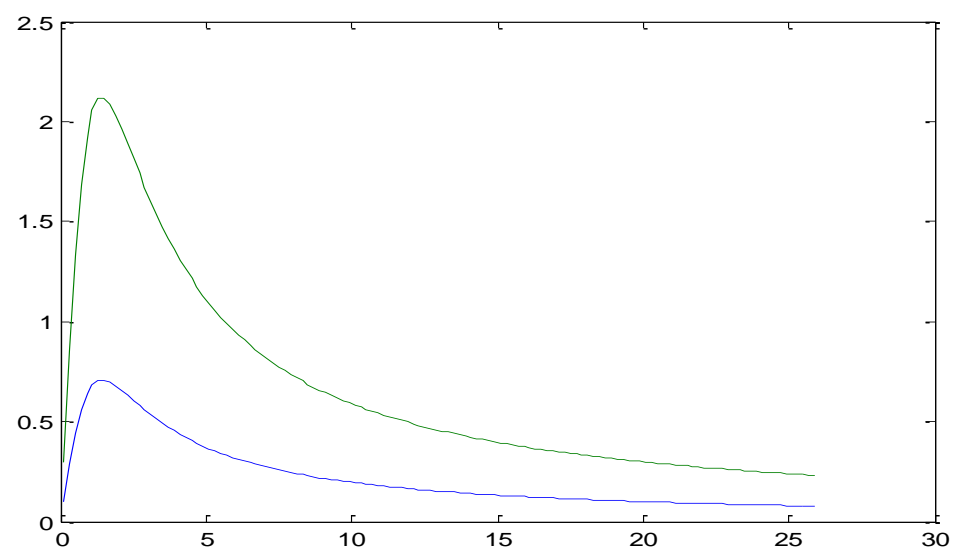

Fig. 2. Hubble's parameter and expansion scalar versus time.

The mean anisotropy parameter and shear scalar are given by

$$
\begin{aligned}
A_{m} & =-1+\frac{3\left(n^{2}+2\right)}{(n+2)\left(t^{2}+a / b\right)}, \\
\sigma^{2} & =\frac{3}{2}\left(\frac{3\left(n^{2}+2\right)}{(n+2)\left(t^{2}+a / b\right)}\right)\left(\frac{t}{b\left(t^{2}+a / b\right)}\right)^{2} .
\end{aligned}
$$

Since, the evolution of the universe starts with constant volume and the rate of expansion is infinite. As $t$ increases, the spatial volume increases but the expansion scalar decreases. Hence, the rate of expansion of the universe decreases as the time increases. When $t \rightarrow \infty$, the spatial volume becomes infinitely large but the expansion stops and 
shear becomes zero. The graphical performance of Hubble parameter is exposed in the Fig. 2.

Fig. 3 demonstrates the behavior of energy density of the universe verses cosmic time $t$ in the evolution of universe as representative case with appropriate choice of constants. It is observed that the energy density of derived model is always positive. At an initial stage $t \rightarrow 0$, energy density is also zero i.e. $\rho \rightarrow 0$, with the expansion for some interval of time it is increases but for hole interval it is decreases up to a small positive value (nearly equal to zero).

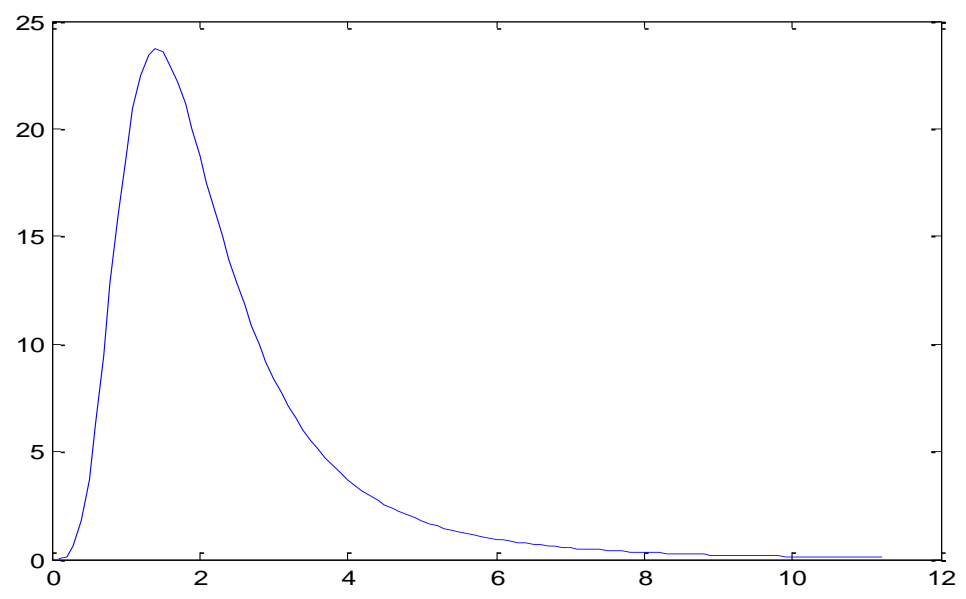

Fig. 3. Energy density versus time.

Coefficient of bulk viscosity

$$
\xi=\frac{-1}{(b k)^{2}(n+2)\left(t^{2}+a / b\right)^{2}}\left\{\alpha_{1}+\frac{\alpha_{2}}{\left(t^{2}+a / b\right)^{2 m-2}}\right\} .
$$

Initially for some interval of time the coefficient of bulk viscosity is negative and increasing but for whole interval which is positive decreasing function of time and final it approaches to a constant quantity which is near to zero. This is in good agreement with the physical behavior of $\xi$ the shown in Fig. 4 . 


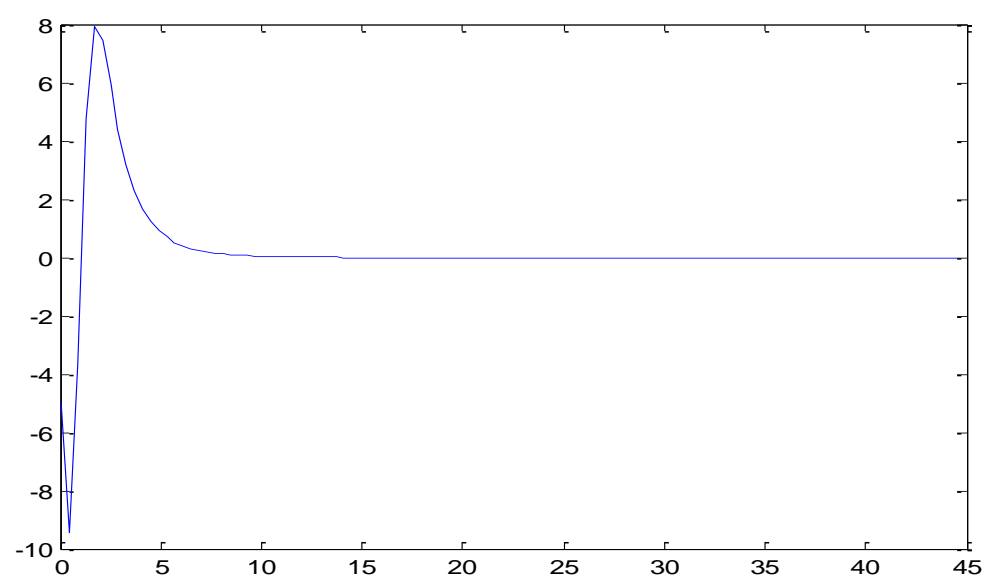

Fig. 4. Bulk viscous coefficient versus time.

\section{Conclusions}

In our investigations we observed that inflation is possible in the universe, the volume starts with a constant volume at $t \rightarrow 0$ and with the stimulation of time it is expanding and increasing, when $t \rightarrow \infty$ the spatial volume $V \rightarrow \infty$. The mean Hubble parameter, expansion scalar are the functions of time and confirmed that the universe is expanding with the increase of cosmic time but the rate of expansion decrease which shows that the universe starts evolving with a constant volume with an infinite rate of expansion. The evolution of the universe starts with constant volume and the rate of expansion is infinite, the spatial volume becomes infinitely large but the expansion stops and shear becomes zero.

It is observed that the energy density of the derived model is always positive as representative case with appropriate choice of constants. At an initial stage $t \rightarrow 0$, energy density is also zero i.e. $\rho \rightarrow 0$, with the expansion for some interval of time it is increases but for hole interval it is decreases up to a small positive value (nearly equal to zero).The coefficient of bulk viscosity spectacles the same deeds as that of the energy density of the Universe.

\section{Acknowledgments}

Authors are grateful to the editor in chief of the journal and anonymous reviewer for fruitful comments on the paper which improved the quality of the paper.

\section{References}

1. E. J. Copeland, M. Sami, and S. Tsujikawa, Int. J. Mod. Phys. D 15, 1753 (2006). https://doi.org/10.1142/S021827180600942X 
2. T. Chiba, T. Kobayashi, M. Yamaguchi, and J. Yokoyama, Phys. Rev. D 75, 043516 (2007). https://doi.org/10.1103/PhysRevD.75.043516

3. S. Nojiri and S. D. Odintsov, Int. J. Geom. Methods Mod. Phys. 4, 115 (2007). https://doi.org/10.1142/S0219887807001928

4. S. Nojiri and S. D. Odintsov, Int. J. Geom. Methods Mod. Phys. 82, 451 (2010).

5. M. Akbar and R. Cai, Phys. Lett. B 635, 7 (2006). https://doi.org/10.1016/j.physletb.2006.02.035

6. T. Harko, F. Lobo, S. Nojiri, and S. Odintsov, Phys. Rev. D 84, 024020 (2011).

7. M. Sharif and M. Zubair, J. Cosmol. Astroparticle Phys. 03, 28 (2012).

8. S. Katore, B. Chopde and S. Shekh, Int. J. Basic Appl. Res. (Special issue) 283 (2012).

9. S. Chandel and S. Ram, Indian J. Phys. 87, 1283 (2013).

10. R. Chaubey and A. Shukla, Astrophys Space Sci. 343, 415 (2013). https://doi.org/10.1007/s10509-012-1204-5

11. P. Sahoo, B. Mishra, G. Chakradhar, and D. Reddy, Eur. Phys. J. Plus 129, 49 (2014). https://doi.org/10.1140/epjp/i2014-14196-9

12. S. R. Bhoyar, V. R. Chirde, and S. H. Shekh, Int. J. Adv. Res. 3, 492 (2015).

13. S. R. Bhoyar, V. R. Chirde, and S. H. Shekh, Int. J. Appl. Res. 6, 398 (2016)

14. V. Chirde and S. Shekh, Astrofisica, 58, 121 (2015).

15. V. R. Chirde and S. H. Shekh, J. Astrophys. Astronomy 37, 15 (2016). https://doi.org/10.1007/s12036-016-9391-z

16. A. Einstein, Phys. Math. Kl. 17, 217 (1928).

17. A. Einstein, Math. Ann. 102, 685 (1930). https://doi.org/10.1007/BF01782370

18. V. R. Chirde, S. H. Shekh, J. Astrophys. Astr. 39, 56 (2018). https://doi.org/10.1007/s12036018-9555-0

19. V. R. Chirde, S. H. Shekh, Ind. J. Phys. 92, 1485 (2018). https://doi.org/10.1007/s12648-0181236-y

20. S. H. Shekh, V. R. Chirde, Gen. Relativ. Gravit. 51, 87 (2019). https://doi.org/10.1007/s10714019-2565-7

21. M. Jamil, D. Momeni, and R. Myrzakulov, Eur. Phys. J. C 72, 2122 (2012). https://doi.org/10.1140/epjc/s10052-012-1998-x

22. M. Setare and M. Houndjo, Can. J. Phys. 91, 168 (2013). https://doi.org/10.1139/cjp-2012-0414

23. V. R. Chirde and S. H. Shekh, Bulgerian J. Phy. 41, 258 (2014).

24. V. R. Chirde and S. H. Shekh, African Rev. Phys. 10, 0020145 (2015).

25. V. R. Chirde and S. H. Shekh, African Rev. Phys. 10, 0057475 (2015).

26. S. R. Bhoyar, V. R. Chirde, and S. H. Shekh, Astrophy. 60 (2), 277 (2017). https://doi.org/10.1007/s10511-017-9480-y

27. S. Aygün, H. Baysal, C. Aktaş, I. Yılmaz, P. K. Sahoo, and I. Tarhan, Int. J. Mod. Phys. A 33, 1850184 (2018). https://doi.org/10.1142/S0217751X18501841

28. A. Vilenkin, Phys. Rep. 121, 263 (1985).

29. Ya. Zel'dovich, B. Mon. Not. R. Astron. Soc. 192, 663 (1980).

30. A. Vilenkin, Phys. Rev. D 24, 2082 (1981).

31. P. S. Letelier, Phys. Rev. D 28, 2414 (1983).

32. P. K. Sahoo, Int. J. Theor. Phys. 49, 25 (2010). https://doi.org/10.1007/s10773-009-0174-z

33. K. L. Mahanta, A. K. Biswal, and P. K. Sahoo, Eur. Phys. J. Plus 129, 141 (2014). https://doi.org/10.1140/epip/i2014-14141-0

34. P. K. Sahoo, A. Nath, and S. K. Sahu, Iran J. Sci. Technol. Trans. Sci. 41, 243 (2017). https://doi.org/10.1007/s40995-017-0214-0

35. N. O. Santos, R. S. Dias, and A. Benerjee, J. Math. Phys. 26, 876 (1985). https://doi.org/10.1063/1.526582

36. X. X. Wang, Astrophys. Space Sci. 298, 433 (2005).

37. M. K. Yadav, A. Rai, and A. Pradhan, Int. J. Theor. Phys. 46, 2677 (2007). https://doi.org/10.1007/s10773-007-9381-7 
38. D. Pavon, J. Bafluy, and D. Jou, Classical Quantum Gravity 8, 347 (1991).

https://doi.org/10.1088/0264-9381/8/2/014

39. R. Maartens, S. D. Maharaj, and B. O. J. Tupper, Classical Quantum Gravity 12, 1455 (1995).

https://doi.org/10.1088/0264-9381/12/10/015 